\title{
Functional relevance of genes implicated by obesity genome-wide association study signals for human adipocyte biology
}

\author{
F. Bernhard • K. Landgraf • N. Klöting • A. Berthold • \\ P. Büttner • D. Friebe • W. Kiess • P. Kovacs • M. Blüher • \\ A. Körner
}

Received: 27 February 2012 / Accepted: 8 October 2012 /Published online: 16 November 2012

(C) Springer-Verlag Berlin Heidelberg 2012

\begin{abstract}
Aims/hypothesis Genome-wide association studies (GWAS) have identified numerous single-nucleotide polymorphisms associated with obesity, consequently implying a role in adipocyte biology for many closely residing genes. We investigated the functional relevance of such genes in human adipocytes.

Methods We selected eight genes (BDNF, MAF, MTCH2, NEGR1, NPC1, PTER, SH2B1 and TMEM18) from obesity GWAS and analysed their effect in human adipogenesis using small interfering (si)RNA-mediated knockdown, their regulation by metabolic agents in adipocytes and preadipocytes, and gene expression in paired samples of human fat biopsies (68 non-obese, 165 obese) by quantitative realtime PCR.

Results We show a two- to threefold upregulation of $M A F$, $M T C H 2$ and NEGR1 and a two- to fourfold downregulation of $B D N F$ and PTER during adipogenesis. Knockdown of
\end{abstract}

F. Bernhard and K. Landgraf contributed equally to this study.

Electronic supplementary material The online version of this article (doi:10.1007/s00125-012-2773-0) contains peer-reviewed but unedited supplementary material, which is available to authorised users.

F. Bernhard · K. Landgraf · A. Berthold · P. Büttner · D. Friebe ·

W. Kiess · A. Körner $(\bowtie)$

Center for Pediatric Research, Hospital for Children \& Adolescents,

Department of Women's and Child Health,

University of Leipzig,

Liebigstraße 21,

04103 Leipzig, Germany

e-mail: antje.koerner@medizin.uni-leipzig.de

K. Landgraf $\cdot$ N. Klöting $\cdot$ M. Blüher $\cdot$ A. Körner

Medical Center Adiposity Diseases (IFB), University of Leipzig,

Leipzig, Germany

N. Klöting $\cdot$ P. Kovacs $\cdot$ M. Blüher

Department of Medicine, University of Leipzig,

Leipzig, Germany
BDNF (mean \pm SEM; $83.8 \pm 4.7 \%$ of control; $p=0.0002$ ), MTCH2 (72.7 $\pm 9.5 \% ; p=0.0006)$, NEGR1 $(70.2 \pm 5.7 \% ; p$ $<0.0001)$ and TMEM18 $(70.8 \pm 6.1 \% ; p<0.0001)$ significantly inhibited adipocyte maturation, while knockdown of the other proteins had no effect. Insulin slightly induced MAF (1.65-fold; $p=0.0009)$ and MTCH2 (1.72-fold; $p<$ $0.0001)$, while it suppressed $B D N F(59.6 \% ; p=0.0009)$, NEGR1 (58.0\%; $p=0.0085)$ and TMEM18 $(69.3 \%$; $p=$ $0.0377)$ in adipocytes. The synthetic glucocorticoid dexamethasone suppressed MAF (45.7\%; $p=0.0022), B D N F$ $(66.6 \% ; p=0.0012)$ and TMEM18 $(63.5 \% ; p=0.0181)$, but induced NEGR1 (3.2-fold; $p=0.0117$ ) expression. Furthermore, MTCH2, NEGR1 and TMEM18 were differentially expressed in subcutaneous and visceral adipose tissue. TMEM18 expression was decreased in the adipose tissue of obese patients, and negatively correlated with anthropometric variables and adipocyte size.

Conclusions/interpretation Our results imply a regulatory role for TMEM18, BDNF, MTCH2 and NEGR1 in adipocyte differentiation and biology. In addition, we show a variation of $M A F$ expression during adipogenesis, while NPC1, PTER and $S H 2 B 1$ were not regulated.

Keywords Adipocyte - Adipogenesis $\cdot B D N F \cdot$ GWAS . MTCH2 $\cdot$ NEGR1 - Obesity · SGBS · TMEM18

$\begin{array}{ll}\text { Abbreviations } \\ \text { BDNF } & \begin{array}{l}\text { Brain-derived neurotrophic factor } \\ \text { CT }\end{array} \\ \begin{array}{ll}\text { Computed tomography } \\ \text { GWAS } & \text { Genome-wide association studies } \\ \text { MAF } & \text { v-Maf musculoaponeurotic fibrosarcoma } \\ & \text { oncogene homologue }\end{array} \\ \text { MTCH2 } & \begin{array}{l}\text { Mitochondrial carrier homologue } 2 \\ \text { NEGR1 }\end{array} \\ \text { Neuronal growth regulator 1 } \\ \text { NPC1 } & \text { Niemann-Pick disease type C1 } \\ \text { PPAR } \gamma & \text { Peroxisome proliferator-activated receptor } \gamma\end{array}$




$\begin{array}{ll}\text { PTER } & \text { Phosphotriesterase-related } \\ \text { SGBS } & \text { Simpson-Golabi-Behmel syndrome } \\ \text { SH2B1 } & \text { SH2B adaptor protein 1 } \\ \text { siRNA } & \text { Small interfering RNA } \\ \text { SNP } & \text { Single-nucleotide polymorphism } \\ \text { TMEM18 } & \text { Transmembrane protein 18 } \\ \text { TSS } & \text { Transcriptional start site }\end{array}$

\section{Introduction}

The accumulation of adipose tissue in obesity results from the storage of excess lipids in the state of positive energy balance. In addition to environmental factors leading to energy overload, genetic predisposition significantly contributes to the susceptibility to obesity. Although the pathogenic mechanisms of rare monogenetic traits are relatively well understood, they constitute only a minor portion of the heritability of obesity. The major share of genetic susceptibility comes from complex polygenic variants.

Genome-wide association studies (GWAS) have identified a large number of single-nucleotide polymorphisms (SNPs) associated with measures of obesity. These include, among other signals, variants in or near the genes $B D N F$ (encoding brain-derived neurotrophic factor), MAF (v-maf musculoaponeurotic fibrosarcoma oncogene homologue), $M T C H 2$ (mitochondrial carrier homologue 2), NEGR1 (neuronal growth regulator 1), $N P C 1$ (Niemann-Pick disease, type C1), PTER (phosphotriesterase-related gene), SH2B1 (SH2B adaptor protein 1) and TMEM18 (transmembrane protein 18) [1-3]. Most of these genes have previously not been recognised as playing a role in the pathogenesis of obesity and a potential role in adipocyte development has not been addressed.

Considering the broad variety of obesity-causing mechanisms that have been described, pathogenic factors might interfere with the regulation of energy expenditure and food intake in the brain, brown adipose tissue-induced energy expenditure [4], oral lipid sensing [5] or gut microbiome composition [6]. In addition, potential causal factors might act on the modification of energy metabolism at the level of adipocytes, or on the formation and expandability of adipose tissue itself [7]. The development of adipose tissue is a highly regulated process based on a complex system of transcription factors and other regulators [7]. Dysregulation in this network can cause obesity by hyperplasia or hypertrophy of adipocytes.

So far, little is known about the potential role of the genes implicated by obesity GWAS signals in adipogenesis and adipocyte biology. In this study, we assessed the functional importance of the respective genes for adipocyte differentiation, their expression during adipocyte differentiation and in human adipose tissue samples, and their transcriptional regulation by metabolic factors affecting lipid metabolism.

\section{Methods}

Cell culture and in vitro adipocyte differentiation We used the human pre-adipocyte model of Simpson-Golabi-Behmel syndrome (SGBS) cells kindly supplied by M. Wabitsch (Division of Pediatric Endocrinology and Diabetes, University of Ulm, Ulm, Germany) [8]. Cells were cultured in basal medium consisting of DMEM/Ham F12 medium (Life Technologies, Karlsruhe, Germany) supplemented with $33 \mu \mathrm{mol} / 1$ biotin and $17 \mu \mathrm{mol} / 1$ pantothenic acid. Preadipocytes were differentiated into mature adipocytes as previously described [9]. Briefly, differentiation was induced under serum-free conditions by treating confluent cells with a medium comprising $20 \mathrm{nmol} / 1$ insulin, $100 \mathrm{nmol} / 1$ hydrocortisone, $0.2 \mathrm{nmol} / 1$ tri-iodothyronine and $0.13 \mathrm{nmol} / 1$ apo-transferrin. For the first 4 days of differentiation, the medium was additionally supplemented with $25 \mathrm{nmol} / \mathrm{l}$ dexamethasone, $500 \mu \mathrm{mol} / 1$ isobutyl-1methylxanthine and $2 \mu \mathrm{mol} / \mathrm{l}$ rosiglitazone.

Stimulation with metabolic regulators For stimulation experiments, confluent pre-adipocytes and mature adipocytes at day 9 post-induction were starved for $24 \mathrm{~h}$ in a serum-free basal medium (DMEM/F12, $33 \mu \mathrm{M}$ biotin and $17 \mu \mathrm{M}$ pantothenic acid) followed by $24 \mathrm{~h}$ incubation with insulin (100 nmol/l), IGF-1 (100 nmol/l) and dexamethasone $(100 \mathrm{nmol} / \mathrm{l})$, or isoprenaline $(10 \mu \mathrm{mol} / \mathrm{l})$. Insulin and IGF-1 are adipogenic agents and promote glucose uptake, fatty acid transport protein translocation and fatty acid uptake in adipocytes $[10,11]$. Dexamethasone is a synthetic glucocorticoid regulating the expression of PPARG and CEBPA, which are involved in adipogenesis [12]. Isoprenaline is a betaadrenergic agonist that induces the lipolytic activity of adipocytes [13]. Untreated cells incubated in a serum-free basal medium served as a control. Four independent cell culture experiments were performed, each in triplicate.

Quantitative real-time PCR Total RNA from cells was extracted using the RNeasy Mini Kit (Qiagen, Hilden, Germany) including on-column DNA digestion according to the manufacturer's instructions. Pooled RNA samples from human tissues were obtained from Clontech-Takara Bio (SaintGermain-en-Laye, France). A total of 500 ng of RNA was reverse transcribed using M-MLV reverse transcriptase and random hexamer $\left[\mathrm{p}(\mathrm{dN})_{6}\right]$ primers. Quantitative real-time TaqMan PCR (Applied Biosystems, Darmstadt, Germany) was performed in a $20 \mu \mathrm{l}$ reaction mix with $10 \mu \mathrm{l} 2 \times \mathrm{qPCR}$ MasterMix Plus Low ROX (Eurogentec, Seraing, Belgium), $900 \mathrm{nmol} / \mathrm{l}$ of each primer and $200 \mathrm{nM}$ probe using the ABI 
7500 Real-Time PCR System (Applied Biosystems). All samples were analysed in triplicate. A standard curve of serial dilutions of plasmid-DNA of the respective target gene was included on each plate. The copy number of each sample was calculated from the standard curve and normalised to the mean of the three housekeeping genes TBP, ACTB and HPRT1. The primer and probe sequences used are listed in the electronic supplementary material (ESM; Table 1). For $S H 2 B 1$, a commercially available gene expression assay was applied (Applied Biosystems). Analyses of candidate gene expression in adipose tissue samples of lean and obese humans were performed as previously described [14].

siRNA-mediated knockdown of candidate proteins SGBS pre-adipocytes were transfected using the Neon Transfection System $100 \mu \mathrm{l}$ Kit (Invitrogen, Carlsbad, CA, USA). Electroporation parameters were optimised to pulse voltage $1300 \mathrm{~V}$, pulse width $20 \mathrm{~ms}$, pulse number 2 and a cell density of $6 \times 10^{6}$ cells/ml. Gene-specific ON-TARGETplus SMARTpool small interfering (si)RNAs and ON-TARGETplus control reagents (Dharmacon, Lafayette, LA, USA), consisting of four different siRNAs for one target, were used at a final concentration of $500 \mathrm{nmol} / \mathrm{l}$. After electroporation, 60,000 cells/well were seeded in 12-well format and differentiated as described above. Efficient knockdown of gene expression was confirmed on days $0,2,4$ and 8 post-induction using quantitative real-time PCR.

Quantification of triacylglycerol contents To confirm and quantify differentiation efficiency, differentiated adipocytes were double-stained with Nile Red and Hoechst 33342 and analysed by fluorescence microscopy. Differentiation efficiency was determined by quantitative morphometric assessment. For quantification of triacylglycerol accumulation, adipocytes at day 8 post-induction were fixed in Roti-Histofix 4\% (Carl Roth GmbH, Karlsruhe, Germany), washed with PBS and stained with Oil Red-O solution ( $0.3 \%$ in $60 \%$ isopropanol) for $15 \mathrm{~min}$. After extensive washing with water, Oil Red-O was extracted by incubation with isopropanol, and quantified at $540 \mathrm{~nm}$ using the FLUOstar OPTIMA (BMG LABTECH, Offenburg, Germany).

Measurement of adipocyte cell size Adipocytes from human adipose tissue samples were isolated by collagenase digestion $(1 \mathrm{mg} / \mathrm{ml})$. To determine cell size distribution, $200 \mu \mathrm{l}$ aliquots of adipocyte suspension were fixed with osmic acid, incubated for $48 \mathrm{~h}$ at $37^{\circ} \mathrm{C}$ and counted in a Coulter counter (Multisizer III; Beckmann Coulter, Krefeld, Germany).

Expression of candidate genes in human adipose tissue samples Paired samples of visceral and subcutaneous adipose tissue were obtained from 233 Europid men $(n=83)$ and women $(n=150)$ who underwent open abdominal surgery for cholecystectomy, appendectomy, weightreduction surgery, abdominal injuries or explorative laparotomy (ESM Table 2). Ages ranged from 18 to 89 years and BMI from 13.8 to $71.0 \mathrm{~kg} / \mathrm{m}^{2}$. A total of 69 participants had type 2 diabetes. All participants had a stable weight without fluctuations of more than $3 \%$ of body weight for at least 3 months before surgery. Patients with severe conditions including generalised inflammation or end-stage malignant diseases were excluded from the study. Samples of visceral and subcutaneous adipose tissue were immediately frozen in liquid nitrogen after explantation. The study was approved by the ethics committee of the University of Leipzig (Leipzig, Germany). All participants gave written informed consent before taking part in the study.

Statistical analyses Statistical analyses of cell culture experiments were performed using GraphPad Prism 4 (GraphPad Software, San Diego, CA, USA). Significance levels were calculated by one-way ANOVA of repeated measurements with Dunnett's post-test and Student's $t$ test. For cell culture analyses, at least three independent experiments were performed, each in triplicate. Differences in gene expression between visceral and subcutaneous adipose tissue were assessed using the paired Student's $t$ test. For statistical analyses on significant differences of mRNA levels in different obesity states, we combined lean and overweight participants into a non-obese/lean subgroup and compared them with the obese subgroup. Linear relationships were assessed by least-square regression analysis using SPSS (Chicago, IL, USA). The associations of the obesity-associated variants with adipose tissue mRNA levels were analysed assuming different genetic models (additive, dominant and recessive) using one-way ANOVA. Twosided $t$ tests were performed and the threshold for statistical significance was set at $p<0.05$.

\section{Results}

Selection of candidate genes Candidate genes for functional analyses were selected from five GWAS [1-3, 15, 16] and a replication study [17] targeting obesity and measures of fat distribution. To prioritise genes with higher potential for clinical and functional relevance for adipogenesis, we applied a score based on significance level, replication of the loci in independent studies, OR, gene-related location of the variant and expression data in human adipocytes (Table 1; ESM Fig. 1). We excluded FTO [18-20] and $M C 4 R$ [21, 22] as potential candidate genes, as previous studies suggested that they mediate their regulatory function by central mechanisms rather than at the level of adipose tissue itself. The top eight genes were selected for further analyses (Table 1). 
Table 1 Selection of candidate genes from GWAS

\begin{tabular}{|c|c|c|c|c|c|c|c|c|c|c|c|c|}
\hline SNP & Nearest gene(s) & $p$ value & Score & Reference & Score & OR & Score & Location & Score & Expression & Score & $\begin{array}{l}\text { Total } \\
\text { score }\end{array}$ \\
\hline rs7498665 & SH2B1 & $5.1 \times 10^{-11}$ & 2 & $2,3,18$ & 1 & 1.1 & 0 & Exonic & 3 & Raw signal $>250 \mathrm{AU}$ & 1 & 7 \\
\hline rs8049439 & $S H 2 B 1$ & $1.4 \times 10^{-9}$ & 2 & 2 & 1 & No data & 0 & Intronic & 2 & Raw signal $>250 \mathrm{AU}$ & 1 & 6 \\
\hline rs 1805081 & $N P C 1$ & $2.9 \times 10^{-7}$ & 1 & 1 & 0 & 0.71 & 1 & Exonic & 3 & Raw signal $>250 \mathrm{AU}$ & 1 & 6 \\
\hline rs6265 & $B D N F$ & $5.1 \times 10^{-10}$ & 2 & 2 & 0 & 1.12 & 0 & Exonic & 3 & Raw signal $>250 \mathrm{AU}$ & 1 & 6 \\
\hline rs 10838738 & MTCH2 & $4.6 \times 10^{-9}$ & 2 & 3 & 1 & No data & 0 & Intronic & 2 & Raw signal $>250 \mathrm{AU}$ & 1 & 6 \\
\hline rs4752856 & MTCH2 & 0.009 & 1 & 18 & 1 & 1.12 & 0 & Intronic & 2 & Raw signal $>250 \mathrm{AU}$ & 1 & 5 \\
\hline rs4788102 & $S H 2 B 1$ & $6.4 \times 10^{-10}$ & 2 & 2 & 1 & No data & 0 & Near gene & 1 & Raw signal $>250 \mathrm{AU}$ & 1 & 5 \\
\hline rs6548238 & TMEM18 & $1.4 \times 10^{-18}$ & 2 & 3 & 1 & 1.1 & 0 & Near gene & 1 & No data & 0 & 4 \\
\hline rs7561317 & TMEM18 & $4.2 \times 10^{-17}$ & 2 & 2 & 1 & 1.2 & 0 & Near gene & 1 & No data & 0 & 4 \\
\hline rs 2867125 & TMEM18 & $1.7 \times 10^{-16}$ & 2 & 2 & 1 & No data & 0 & Near gene & 1 & No data & 0 & 4 \\
\hline rs4854344 & TMEM18 & $6.8 \times 10^{-17}$ & 2 & 2 & 1 & No data & 0 & Near gene & 1 & No data & 0 & 4 \\
\hline rs10508503 & PTER & $2.1 \times 10^{-7}$ & 1 & 1 & 0 & 0.68 & 1 & Near gene & 1 & Cycle threshold $<35$ & 1 & 4 \\
\hline rs 2568958 & NEGR1 & $1.2 \times 10^{-11}$ & 2 & 2 & 1 & 1.07 & 0 & Near gene & 1 & No data & 0 & 4 \\
\hline rs 3101336 & NEGRI & $2.5 \times 10^{-11}$ & 2 & 2 & 1 & No data & 0 & Near gene & 1 & No data & 0 & 4 \\
\hline rs 1424233 & $M A F$ & $3.8 \times 10^{-13}$ & 2 & 1 & 0 & 1.13 & 0 & Near gene & 1 & Cycle threshold $<35$ & 1 & 4 \\
\hline rs4074134 & $B D N F$ & $4.4 \times 10^{-11}$ & 2 & 2 & 0 & No data & 0 & Near gene & 1 & Raw signal $>250 \mathrm{AU}$ & 1 & 4 \\
\hline rs4923461 & $B D N F$ & $3.2 \times 10^{-11}$ & 2 & 2 & 0 & No data & 0 & Near gene & 1 & Raw signal $>250 \mathrm{AU}$ & 1 & 4 \\
\hline rs10501087 & $B D N F$ & $8.7 \times 10^{-11}$ & 2 & 2 & 0 & No data & 0 & Near gene & 1 & Raw signal $>250 \mathrm{AU}$ & 1 & 4 \\
\hline rs1077393 & NCR3, AIF1 & $4.4 \times 10^{-8}$ & 2 & 2 & 0 & No data & 0 & Intronic & 2 & Raw signal $<250 \mathrm{AU}$ & 0 & 4 \\
\hline rs 7826222 & MSRA & $1.2 \times 10^{-8}$ & 2 & 17 & 0 & No data & 0 & Near gene & 1 & Raw signal $>250 \mathrm{AU}$ & 1 & 4 \\
\hline rs11084753 & KCTD15 & $2.3 \times 10^{-8}$ & 2 & 3 & 1 & 1.05 & 0 & Near gene & 1 & Raw signal $<250 \mathrm{AU}$ & 0 & 4 \\
\hline rs29941 & CHST8, KCTD15 & $7.3 \times 10^{-12}$ & 2 & 2 & 1 & 1.1 & 0 & Near gene & 1 & Raw signal $<250 \mathrm{AU}$ & 0 & 4 \\
\hline rs10938397 & GNPDA2 & $3.4 \times 10^{-16}$ & 2 & 3,18 & 1 & 1.1 & 0 & Near gene & 1 & No data & 0 & 4 \\
\hline rs 987237 & TFAP $2 B$ & $4.5 \times 10^{-9}$ & 2 & 17 & 0 & No data & 0 & Intronic & 2 & No data & 0 & 4 \\
\hline rs925946 & $B D N F, A S 1$ & $8.5 \times 10^{-10}$ & 2 & 2 & 0 & 1.11 & 0 & Intronic & 2 & No data & 0 & 4 \\
\hline rs10913469 & $S E C 16 B, R A S A L 2$ & $6.2 \times 10^{-8}$ & 1 & 2 & 0 & 1.1 & 0 & Intronic & 2 & No data & 0 & 3 \\
\hline rs 2815752 & $N E G R 1$ & $6.0 \times 10^{-8}$ & 1 & 3,18 & 1 & 1.1 & 0 & Near gene & 1 & No data & 0 & 3 \\
\hline rs 2260000 & NCR3, AIF1 & $1.4 \times 10^{-7}$ & 1 & 2 & 0 & No data & 0 & Intronic & 2 & Raw signal $<250 \mathrm{AU}$ & 0 & 3 \\
\hline rs7647305 & $\begin{array}{l}\text { SFRS10, ETV5, } \\
\text { DGKG }\end{array}$ & $7.2 \times 10^{-11}$ & 2 & 2 & 0 & 1.11 & 0 & Near gene & 1 & Raw signal $<250 \mathrm{AU}$ & 0 & 3 \\
\hline rs10146997 & $N R X N 3$ & $5.3 \times 10^{-8}$ & 1 & 16 & 0 & No data & 0 & Intronic & 2 & No data & 0 & 3 \\
\hline rs 2844479 & NCR3, AIF1 & $9.0 \times 10^{-8}$ & 1 & 2 & 0 & 1.07 & 0 & Near gene & 1 & Raw signal $<250 \mathrm{AU}$ & 0 & 2 \\
\hline rs 2605100 & LYPLAL1 & $2.5 \times 10^{-8}$ & 1 & 17 & 0 & No data & 0 & Near gene & 1 & No data & 0 & 2 \\
\hline rs7138803 & BCDIN3D, FAIM2 & $1.2 \times 10^{-7}$ & 1 & 2 & 0 & 1.14 & 0 & Near gene & 1 & Raw signal $<250 \mathrm{AU}$ & 0 & 2 \\
\hline
\end{tabular}

All significantly associated variants from GWAS were allocated 1 point, with SNPs reaching genome-wide significance $\left(p<5 \times 10^{-8}\right)$ allocated 2 points. Further scoring criteria were: replication in an independent study (1 point), above-average effect size of the variant with the obesity-related trait (OR $<0.8$ or $>1.2 ; 1$ point), location of the SNP (1 point for intergenic, 2 points for intronic, 3 points for exonic) and expression in differentiating SGBS cells (1 point). Positive expression was defined as a raw signal $>250$ in whole-genome expression data or a cycle threshold value $<35$ in quantitative real-time PCR in the case of MAF and PTER. The top eight genes were selected for in vitro studies. Among the variants reaching a score of overall 4 points, MAF and PTER presented the strongest ORs and were therefore chosen for further analyses. AU, arbitrary units

To test whether the selected candidate genes are indeed associated with the obesity-related genetic variant, we checked the location and functional relevance of any SNP within $200 \mathrm{~kb}$ in linkage disequilibrium with the SNP included in our candidate gene selection procedure $\left(r^{2}>0.8\right.$, HapMap CEU population). Applying our scoring strategy, the candidate genes included in our study were confirmed as the most functionally relevant.
Human tissue expression profile of candidate genes We first investigated the expression level of each of the candidate genes in human adipose tissue and compared it with several metabolic, neural, lymphatic and other tissues. Each of the candidate genes showed a distinct expression profile among human tissues, with no uniform expression pattern for the different genes. All of the genes were expressed in adipose tissue. Only SH2B1 
adipose tissue expression was below the overall median expression of all tissues (Fig. 1).

Functional relevance of candidate gene expression for adipogenesis To investigate whether expression of the candidate genes from GWAS might be essential for adipogenesis, we suppressed candidate protein production in pre-adipocytes using gene-specific siRNAs and assessed the effect on adipocyte differentiation and triacylglycerol accumulation.

Knockdown of peroxisome proliferator-activated receptor $\gamma(\operatorname{PPAR} \gamma)$, a master regulator of adipogenesis [23], resulted in an expected significant reduction in triacylglycerol accumulation $(p<0.0001)$ and served as a positive control (Fig. 2a, b).

Knockdown of BDNF led to a slight but significant reduction in adipogenesis $(p=0.0002)$, while we observed stronger effects after knockdown of MTCH2 $(p=0.0006)$, NEGR1 $(p<0.0001)$, and TMEM18 $(p<0.0001)$. In

Fig. 1 Expression profiles of the candidate genes across human tissues. (a) $B D N F$, (b) $M A F$, (c) $M T C H 2$, (d) NEGR1, (e) $N P C 1$, (f) PTER, (g) $S H 2 B 1$ and (h) TMEM18. The relative mRNA expression of each candidate gene was quantified in a human cDNA tissue panel. Expression levels are standardised to the median expression across all tissues (dotted line). Data are mean \pm SEM
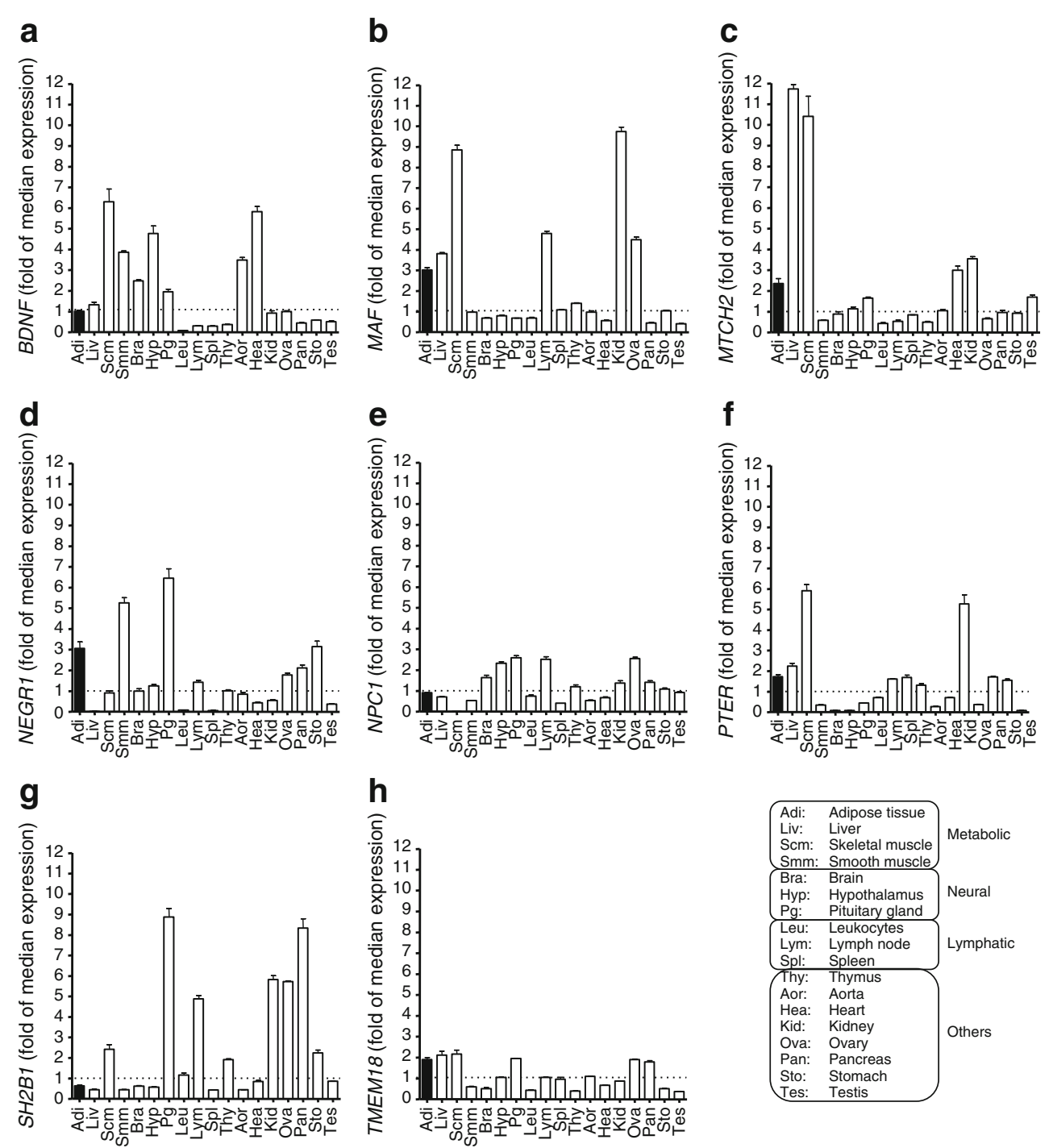

contrast, we did not detect alterations in adipogenesis after knockdown of MAF, NPC1, PTER or SH2B1 production (Fig. 2a, b). Successful knockdown of target gene expression was confirmed by quantitative real-time PCR. Expression of each candidate gene was decreased to at least $60 \%$. All genes were downregulated over the whole differentiation period except for TMEM18 expression, which recovered at day 8 (Fig. 2c).

Expression of candidate genes during human adipocyte differentiation We next evaluated whether candidate gene expression is regulated during human adipocyte differentiation. Efficient adipocyte differentiation was confirmed by Nile Red/Hoechst double staining and fluorescence microscopy (Fig. 3a), and a significant increase in PPARG expression $(p<0.0001)$ (Fig. 3b).

MAF $(p=0.008)$, MTCH2 $(p<0.001)$ and NEGR1 $(p<$ 0.001 ) expression increased at least twofold in mature adipocytes compared with undifferentiated pre-adipocytes. 


\section{a}
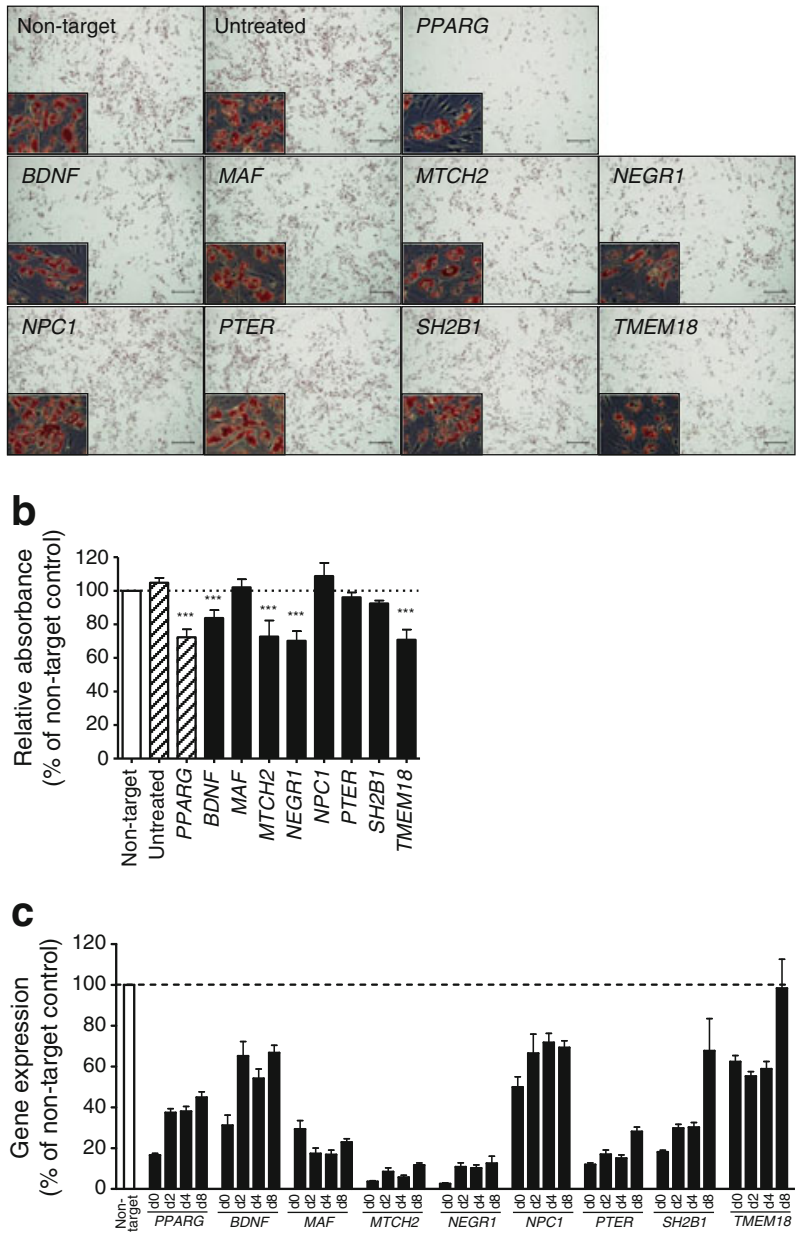

Fig. 2 Effect of knockdown of candidate protein production on adipocyte formation. Pre-adipocytes were transfected with siRNA directed against the respective target gene. (a) Adipocyte differentiation was induced and cells were stained with Oil Red-O at day 8. (b) Triacylglycerol accumulation was quantified by absorbance measurement of the extracted dye, setting the non-target control to $100 \%$. Cells transfected with non-target control siRNA did not differ from untreated cells in terms of adipogenic potential; $* * * p<0.001$. (c) Knockdown efficiency was determined from the day of induction (d0) to day $8(\mathrm{~d} 8)$ of adipocyte differentiation. Data are given as mean \pm SEM. Three independent experiments were performed in triplicate

$B D N F$ expression decreased to about $25 \%(p<0.001)$ and PTER to about $50 \%(p=0.010)$ of their initial expression level (Fig. 3c). In contrast, NPC1 ( $p=0.007)$, and TMEM18 $(p=0.003)$ expression levels were significantly but only slightly modulated (less than twofold) during human adipocyte differentiation, while $S H 2 B 1$ was not significantly altered ( $p>0.05$; data not shown).

Effect of metabolic stimuli on candidate gene expression To analyse the impact of metabolic regulators on candidate genes, we determined the effect of insulin, dexamethasone, IGF-1 and isoprenaline on candidate gene expression in pre-adipocytes and mature adipocytes (Fig. 4).
After incubation of pre-adipocytes with these metabolic agents, we observed no or only modest alterations in the expression levels of the candidate genes, except for $M A F$ expression, which was significantly downregulated by dexamethasone $(p=0.0048)$ and IGF-1 $(p=0.0213)$.

In mature adipocytes, insulin slightly induced expression of $M A F$ and $M T C H 2$, while both insulin and IGF-1 suppressed $B D N F, N E G R 1$ and TMEM18 expression. Dexamethasone significantly inhibited $B D N F(p=0.0012), M A F$ $(p=0.0010)$ and TMEM18 $(p=0.0181)$, but induced NEGRI $(p=0.0117)$ expression levels.

In contrast, the beta-adrenergic agonist isoprenaline did not exert any effect on candidate gene expression in preadipocytes or adipocytes. The expression of NPC1, PTER and $S H 2 B 1$ was not affected by any of the metabolic factors.

Expression in subcutaneous and visceral adipose tissue of lean and obese human participants We investigated the expression of the candidate genes in visceral and subcutaneous human adipose tissue samples from the previously described male $(n=83)$ and female $(n=150)$ donors who underwent abdominal surgery [24]. Further clinical characteristics of the cohort are given in ESM Table 2.

MTCH2 expression in subcutaneous adipose tissue exceeded its expression in visceral adipose tissue $(p<0.01)$, while TMEM18 $(p<0.01)$ and NEGR1 $(p<0.01)$ expression was higher in visceral adipose tissue; there were no differences for $B D N F$ (Fig. 5). Furthermore, $M A F$ expression was significantly lower in subcutaneous compared with visceral adipose tissue $(p<0.0001)$ and $N P C 1$ expression was slightly increased $(p<0.05)$, while PTER and SH2B1 were not differentially expressed (ESM Fig. 2a, d, g, j).

When we stratified the patients into non-obese (BMI $<30 \mathrm{~kg} / \mathrm{m}^{2}$ ) and obese (BMI $\geq 30 \mathrm{~kg} / \mathrm{m}^{2}$ ) groups, TMEM18 expression was lower in both fat depots of obese compared with non-obese participants. Furthermore, MTCH2 expression was significantly increased $(p<0.05)$ in subcutaneous adipose tissue of obese participants, while NEGRI expression was significantly decreased $(p<0.01)$ compared with non-obese participants (Fig. 5). In addition, PTER, but not $M A F, N P C 1$ or $S H 2 B 1$ expression, was significantly associated with the degree of obesity, as detected by a slight decrease in subcutaneous adipose tissue expression levels of obese participants ( $p<0.05$; ESM Fig. 2).

Finally, we analysed potential associations of visceral and subcutaneous candidate gene expression with anthropometric, metabolic and inflammatory variables (Table 2). Corresponding to the decrease in the non-obese group, the expression of TMEM18 in subcutaneous adipose tissue was significantly negatively associated with all clinical variables of body fat mass (weight, BMI, waist and hip circumference, WHR, percent body fat, visceral and subcutaneous fat area, computed 
a

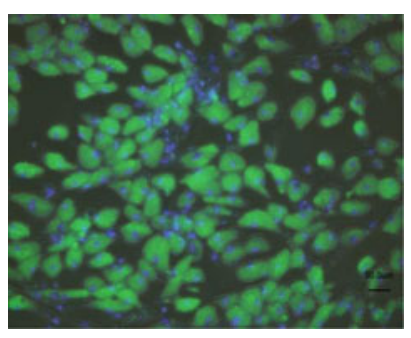

b

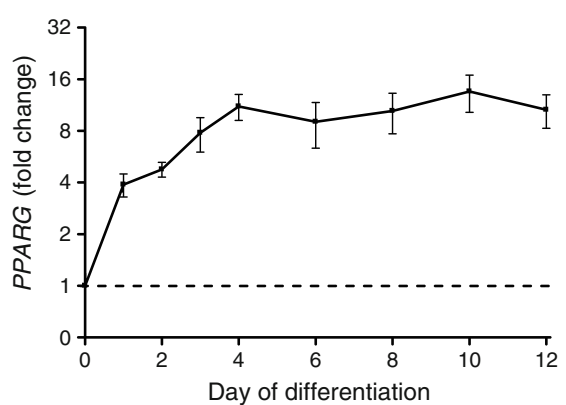

C

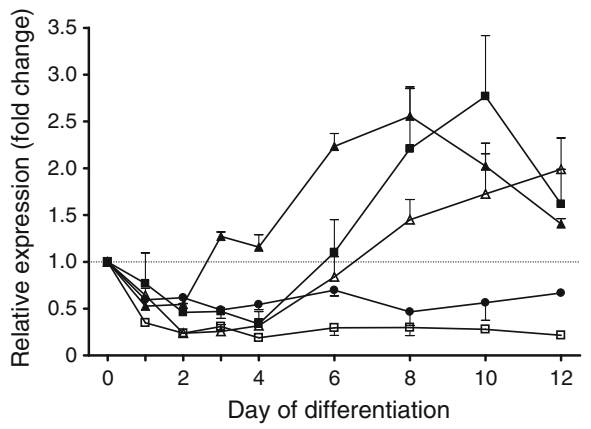

Fig. 3 Expression of candidate genes during human adipogenesis. SGBS pre-adipocytes were differentiated into mature adipocytes over a period of 12 days. (a) Successful adipocyte differentiation was confirmed by Nile Red/Hoechst 33342 staining and (b) the quantification of PPARG mRNA expression. (c) $M A F$ (filled squares, $p=0.008$ ), $M T C H 2$ (filled triangles, $p<0.001$ ) and NEGR1 (empty triangles, $p<$ 0.001 ) were upregulated and $B D N F$ (empty squares, $p<0.001$ ) and

tomography (CT) ratio, serum leptin) and subcutaneous adipocyte size. Along with this, we identified negative correlations between TMEM18 expression and variables of insulin $\left(\mathrm{HbA}_{1 \mathrm{c}}\right.$, fasting plasma insulin) and lipid metabolism (HDL-cholesterol,
PTER (filled circles, $p=0.010$ ) were downregulated by more than twofold during adipocyte differentiation. Three independent experiments were performed in triplicate. Basal expression in untreated preadipocytes was set to 1 and data are given as fold change compared with basal as mean \pm SEM. Expression during adipocyte differentiation was analysed using one-way ANOVA of repeated measurements

triacylglycerol, NEFA) that were small in magnitude but significant. Similarly, $B D N F$ expression in subcutaneous but not in visceral adipose tissue was significantly negatively related to anthropometric variables (weight, BMI, waist and hip
Fig. 4 Effect of metabolic regulators on candidate gene expression. (a) $B D N F$, (b) $M A F$, (c) $M T C H 2$, (d) NEGR1, (e) $N P C 1$, (f) PTER, (g) SH2B1 and (h) TMEM18. After $24 \mathrm{~h}$ starvation, pre-adipocytes and adipocytes on day 10 of differentiation were stimulated with $100 \mathrm{nmol} / \mathrm{l}$ insulin (Ins), $100 \mathrm{nmol} / 1$ dexamethasone (Dex), $100 \mathrm{nmol} / 1 \mathrm{IGF}-1$ or $10 \mu \mathrm{mol} / 1$ isoprenaline (Iso) for $24 \mathrm{~h}$. Untreated cells served as controls (C) and were set to 1 . Data are shown as mean \pm SEM for four independent cell experiments performed in triplicate. Statistical significance was assessed by Student's $t$ test. $* p<0.05 ; * * p<0.01$; $* * * p<0.001$ a

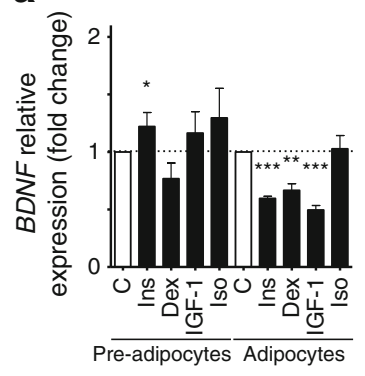

d

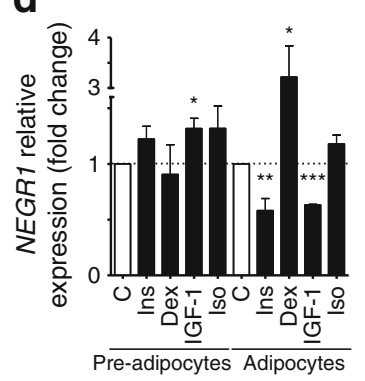

g

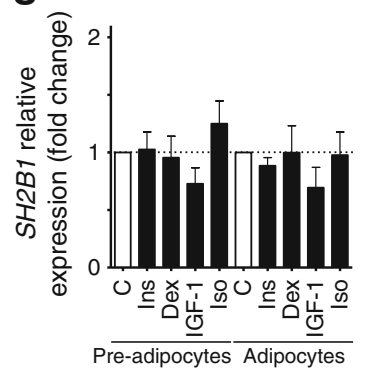

b

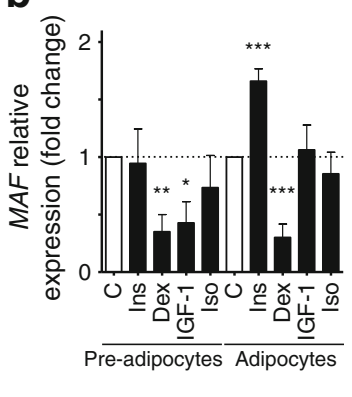

e

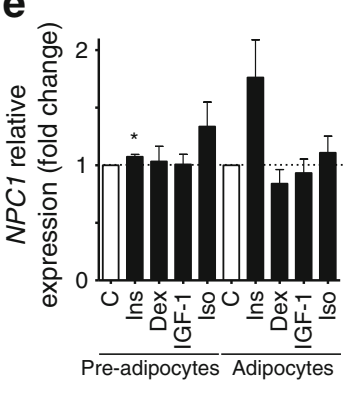

h

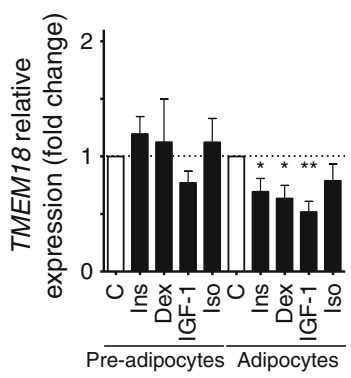

C

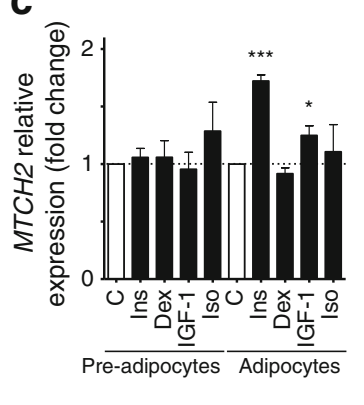

f

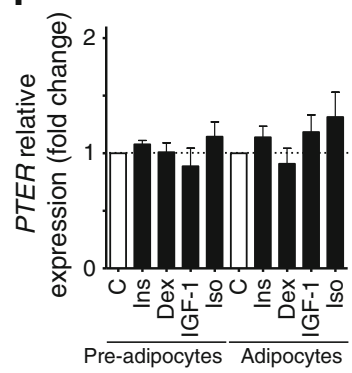


Fig. 5 Expression of $B D N F$, MTCH2, NEGR1 and TMEM18 in adipose tissue of lean and obese humans. Gene expression of $(\mathbf{a}-\mathbf{c}) B D N F,(\mathbf{d}-\mathbf{f}) \mathrm{MTCH} 2$, (g-i) NEGRI and $(\mathbf{j}-\mathbf{l})$ TMEM18 was quantified in paired visceral and subcutaneous samples from 233 donors and normalised to expression of HPRT1. (a, d, g, j) White bars, expression in visceral adipose tissue; black bars, expression in subcutaneous adipose tissue. Data are given as mean \pm SEM and statistical significance was calculated using Student's $t$ test. ${ }^{*} p<0.05 ; * * p<0.01$; $* * * p<0.001$. AU, arbitrary units a

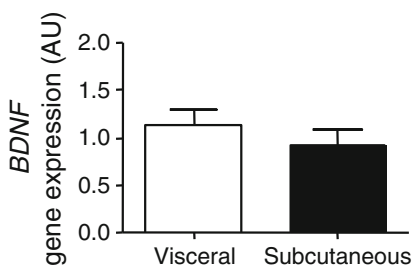

b

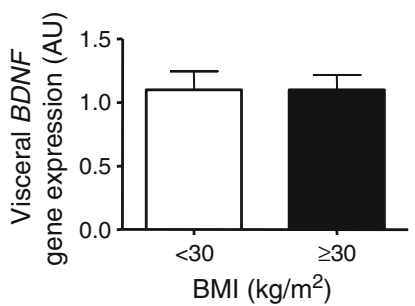

d

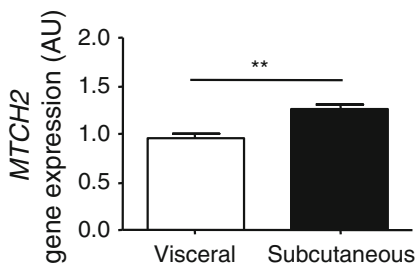

g

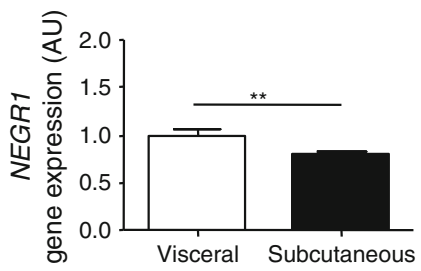

j

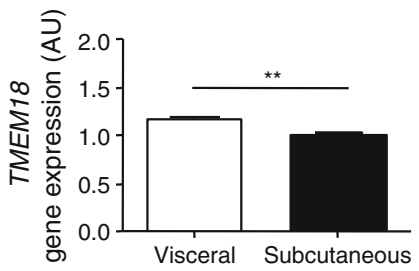

e

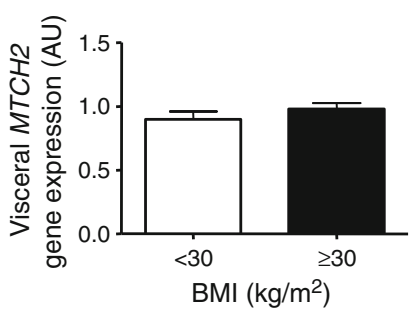

h

k

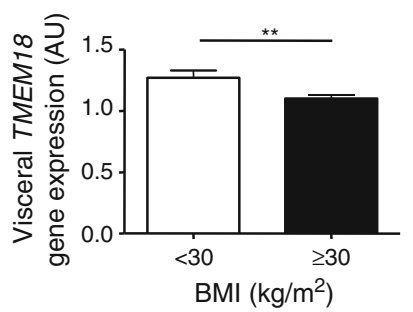

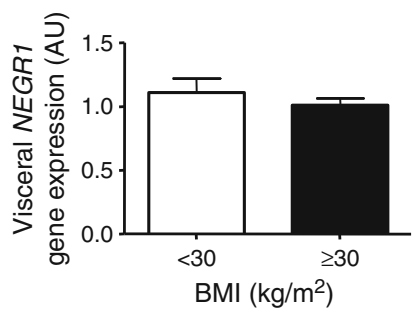

C

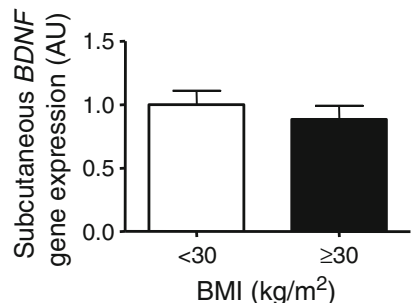

f

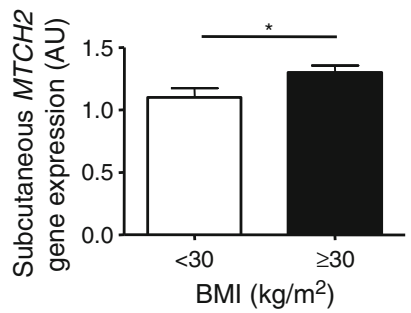

i

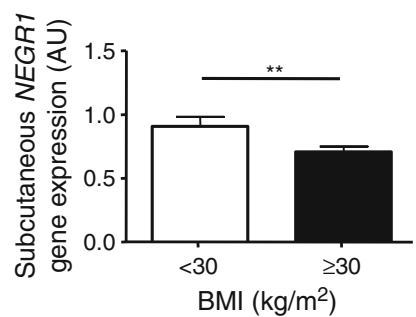

I

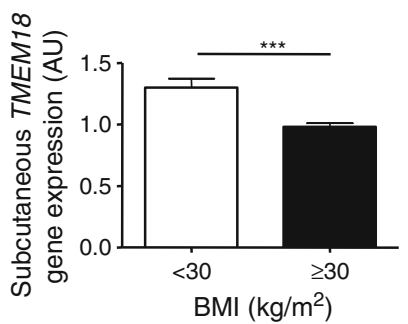

circumference, percent body fat, visceral and subcutaneous fat area, serum leptin) and metabolic variables $\left(\mathrm{HbA}_{1 \mathrm{c}}\right.$, clamp, HDL-cholesterol, triacylglycerol, NEFA). There were some associations with obesity variables (WHR, waist and hip circumference, visceral and subcutaneous fat area) for NEGR1 expression in subcutaneous adipose tissue, but not to the extent observed for TMEM18 or BDNF (Table 2). MTCH2 expression was not associated with clinical variables in adipose tissue (Table 2). Furthermore, we observed slight negative associations of subcutaneous MAF, SH2B1 and PTER expression levels with the degree of obesity (weight, BMI, waist and hip circumference, visceral and subcutaneous fat area, serum leptin concentration) and total cholesterol and LDL-cholesterol as variables of lipid metabolism (ESM Table 3).

Finally, we evaluated whether candidate expression in adipose tissue samples may be affected by the presence of genetic risk alleles. However, there were no significant differences in candidate gene expression between carriers and non-carriers of the risk alleles (ESM Table 4).

\section{Discussion}

In this study, we systematically characterised the functional relevance of genes implicated by obesity GWAS signals at the level of adipocytes in vitro. Our results point to a potential role for TMEM18, BDNF, MTCH2 and NEGR1 as regulators of human adipocyte differentiation.

Of the obesity genes from GWAS investigated in this study, we observed the strongest effects for TMEM18 and BDNF. TMEM18 has been suggested to act as a sequence-specific transcription factor involved in the modulation of neural stem cell migration [25]. Our results, showing differential expression of TMEM18 between the adipose tissue of obese and lean 


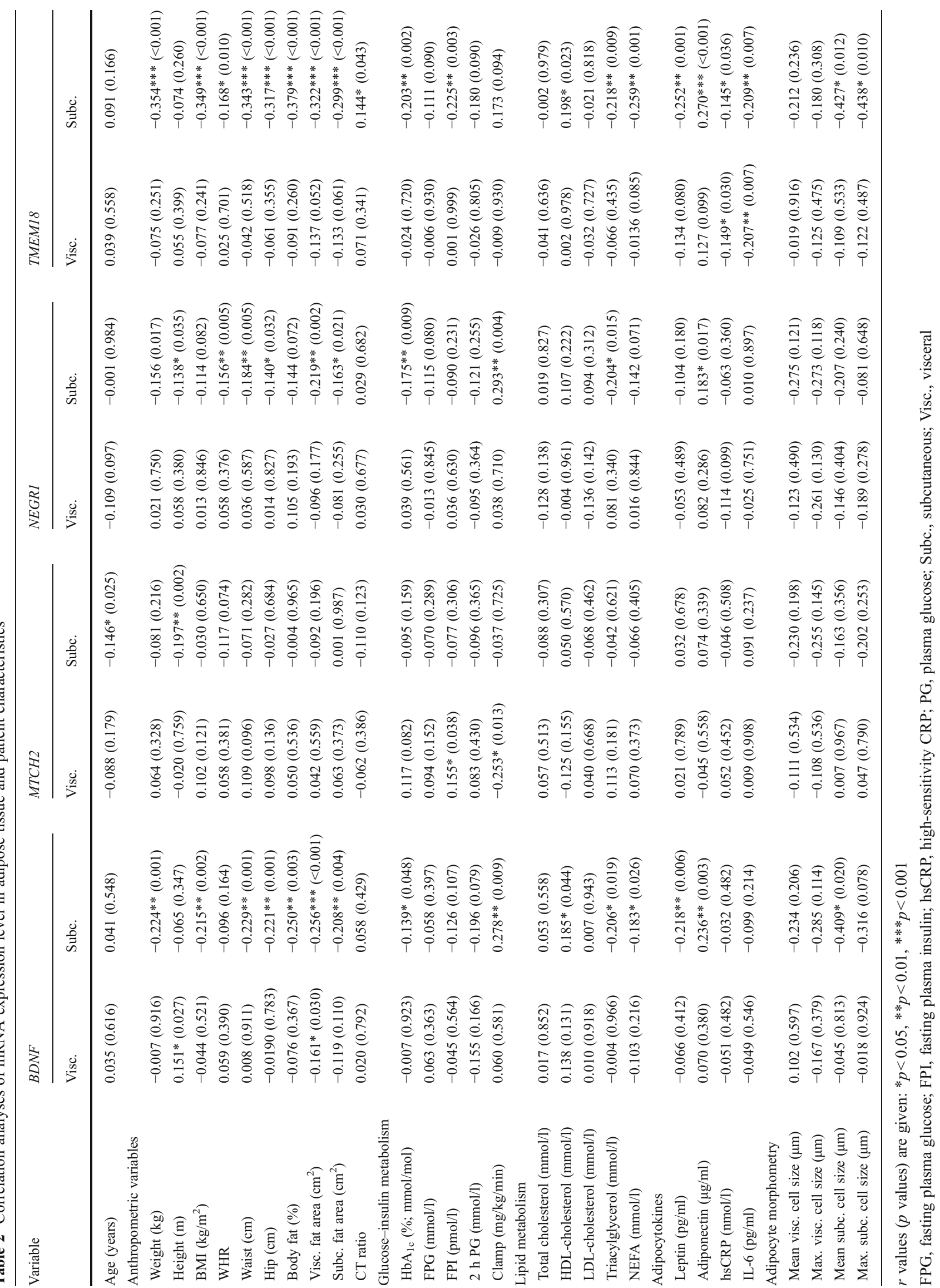


participants and negative regulation of TMEM18 by adipogenic regulators, indicate a role for TMEM18 in adipose tissue. Furthermore, we provide evidence that TMEM18 gene function is essential for adipogenesis since even incomplete suppression of TMEM18 mRNA levels led to significant inhibition of adipocyte differentiation. It is therefore tempting to speculate that TMEM18 acts as a transcriptional regulator in adipogenesis, although targets of TMEM18 in adipose tissue are so far unknown. Interestingly, recent metaanalyses of several GWAS have confirmed the strong association of variations in the TMEM18 locus with measures of obesity. In fact, variants in TMEM18 were the strongest predictors of obesity besides variants in the FTO gene [26, 27].

BDNF has mainly been associated with central neurological and psychiatric disorders [28]. The association of circulating BDNF and genetic BDNF variants with obesity has previously been shown, but is mainly attributed to the central regulation of food intake and energy expenditure (reviewed in [29]). BDNF has been proposed to act downstream of the melanocortin 4 receptor [30], and mice depleted of $B d n f$ in the hypothalamus developed hyperphagic behaviour and obesity [31]. Interestingly, we also detected $B D N F$ expression in visceral and subcutaneous adipose tissue of human participants. $B D N F$ expression levels in subcutaneous adipose tissue were negatively associated with obesity-related variables. In line with these data, previous studies have shown that circulating BDNF levels are lower in children and adolescents with obesity [32], and low levels of BDNF accompanied impaired glucose metabolism in adults [33]. Complementing these clinical observations, $B D N F$ expression was impressively downregulated with induction of adipocyte differentiation in our study. On the other hand, in adipose-derived stem cells treated with a neurogenic induction protocol, $B D N F$ expression was increased [34]. This may indicate a role for BDNF in the commitment of cell lineage determination. Our results provide the first evidence that BDNF is of functional relevance at the level of adipose tissue.

MTCH2 encodes a surface-exposed outer mitochondrial membrane protein that is hypothesised to be involved in apoptosis [35]. A recent study suggested an association between $\mathrm{MTCH} 2$ expression in adipose tissue and obesity in adults [36]. It was shown that MTCH2 mRNA and protein expression were increased in the adipose tissue of obese women and with the progress of adipocyte differentiation in vitro [36]. In line with this study, we detected an induction of $\mathrm{MTCH} 2$ in mature adipocytes compared with preadipocytes and differential expression of $\mathrm{MTCH} 2$ in the subcutaneous adipose tissue of non-obese and obese human participants, indicating a role for $\mathrm{MTCH} 2$ in adipose tissue biology. This corresponds with recent results showing that MTCH2 was upregulated in the adipose tissue of rats exposed to a high-fat diet [37]. The upregulation of $\mathrm{MTCH} 2$ expression during adipogenesis and in the adipose tissue of obese humans is similar to previous results obtained for expression of PPARG, which is activated with the start of adipogenesis and increased in the adipose tissue of obese humans $[38,39] . P P A R G$ is a transcription factor and a key regulator of adipocyte differentiation [7]. One might therefore speculate that $M T C H 2$ is involved in the adipogenic programme that is triggered upon $P P A R G$ activation.

NEGR1 was originally identified as a neural protein with high expression in the cerebral cortex and hippocampus [40]. It was postulated to be involved in synaptic formation during neuronal development and, hence, in plasticity in learning and memory [41]. Interestingly, $N E G R 1$ was recently shown to be differentially regulated in adipose tissue of lean and obese siblings [42]. Similarly, we detected lower NEGR1 expression in the subcutaneous adipose tissue of obese human participants and observed slight but significant negative associations between subcutaneous NEGR1 expression and obesity-related variables. These findings might indicate a possible regulatory function of NEGR1 in adipose tissue.

MAF has been described as a transcription factor involved in the commitment of mesenchymal stem cells into the osteoblast or adipocyte lineage [43]. We did not observe an effect on adipocyte differentiation in the absence of $M A F$ expression, which might be due to the fact that the SGBS preadipocyte model used in this study is already committed to the adipocyte lineage and does not represent mesenchymal stem cells. However, the differential expression of MAF during pre-adipocyte/adipocyte transition and the modulation by metabolic agents involved in adipocyte differentiation may point to a role for $M A F$ in adipocyte differentiation.

Finally, our results do not suggest a role for $\mathrm{NPC1}, \mathrm{SH} 2 \mathrm{B1}$ or PTER at the level of adipose tissue. There might be distinct mechanisms by which these genes are involved in the accumulation of fat mass, as is the case for the FTO gene. Variants in the $F T O$ gene show the strongest associations with obesity and metabolic traits so far [27]. Several clinical and experimental studies indicate that FTO mediates a regulatory function by central mechanisms, rather than at the level of adipose tissue itself [18-20]. Similarly, $S H 2 B 1$ was previously described as a central regulator of weight control [44]. Sh2b1 knockout resulted in morbid obesity and type 2 diabetes in mice, which was explained by an enhancement of hypothalamic leptin sensitivity $[45,46]$. The NPC1 gene codes for a protein involved in intracellular cholesterol trafficking and is primarily known for an autosomal-recessive lysosomal lipid storage disorder characterised by cellular lipid accumulation, neurodegeneration and reduced steroid production [47]. In a recent study, decreased $\mathrm{NPCl}$ gene dosage made mice susceptible to obesity and insulin resistance, but the mechanisms behind this remain unclear [48]. One has to note that in our study, siRNA-mediated knockdown of NPC1 led to a reduction of $\mathrm{NPCl}$ expression by about $50 \%$, hence making it difficult to completely rule out the relevance of NPC1 for adipogenesis of SGBS cells. Of note, the 
obesity-associated variants mapping to NPC1 and PTER were not replicated in independent studies and did not achieve the high significance levels in GWAS compared with variants in other candidate genes.

We did not detect a significant association between risk alleles and the expression level in adipose tissue for any of the genes analysed. However, this may be explained by insufficient statistical power due to small sample size.

Concerning the selected genes for these functional analyses, we need to remember that the selection procedure used in this study was subjective, even though a scoring approach was applied. For example, the location of a variant with respect to the transcriptional start site (TSS) might be an important selection criterion, since previous studies have suggested that expression quantitative trait loci are enriched within $200 \mathrm{~kb}$ of a TSS [49]. If we considered variants near TSSs as functionally relevant as exonic variants and awarded equal points for each, the top eight candidate genes would remain the same with one exception. The SNP rs6747305 is located near the genes ETV5, SFRS10 and DGKG, most closely mapping to ETV5. Interestingly, ETV5 encodes a transcription factor and promotes adipogenesis in 3T3-L1 cells [50]. Furthermore, the number of GWAS, meta-analyses and replication studies has been steadily increasing since the initiation of this project, implicating additional potential candidate genes. In addition, although our analyses support the candidate gene selection, we cannot be certain that the genes we selected represent the causal genes underlying the association of the respective variants with obesity-related traits. Nevertheless, the systematic analyses of those genes are of great importance to preselect genes with a functional relevance during adipogenesis in vitro.

In summary, we have shown functional implications for TMEM18, BDNF, MTCH2 and NEGR1 during adipogenesis. Hence, the obesity-associated GWAS signals at the TMEM18, BDNF, MTCH2 and NEGR1 loci might be explained by effects on gene expression and subsequent gene function in adipose tissue. In addition, $M A F$ expression is differentially regulated in adipocytes but is not essential for adipogenesis. NPC1, PTER and SH2B1 did not have a regulatory role at the level of adipose tissue in our study.

Acknowledgements This work was supported by grants from the Medical Faculty, University of Leipzig, to F. Bernhard, the German Research Council DFG, KFO 152 'Atherobesity' (KO3512/2-1) to A. Körner, the Federal Ministry of Education and Research (BMBF), Germany, FKZ: 01EO1001 (IFB Adiposity Diseases ADI K7-10 to A. Körner, 50B to N. Klöting and ADI K7-30 to M. Blüher) and the European Community's 7th Framework Programme (FP7/2007-2013) under grant agreement $n^{\circ} 279153$ 'Beta-JUDO' to A. Körner. P. Kovacs is funded by the Boehringer Ingelheim Foundation. SGBS cells were kindly provided by M. Wabitsch (Ulm, Germany). We thank D. Petroff (Coordination Center for Clinical Trials, University of Leipzig, Leipzig, Germany) for revising the manuscript for language and grammar.

Duality of interest The authors declare that there is no duality of interest associated with this manuscript.
Author contributions FB researched the majority of data (cell culture, tissue arrays, expression analyses and knockdown) and wrote the manuscript draft. KL designed and supervised experiments, analysed and interpreted data, wrote the manuscript and contributed to discussions. NK researched and statistically analysed the data (tissue sample expression). AB researched data (cell culture, expression analyses). $\mathrm{PB}$ researched data (quantitative expression analyses). DF researched data (standard plasmids). WK interpreted data and contributed to discussions. PK researched data (genetic analyses) and contributed to discussions. MB researched data (tissue biology and patient phenotyping) and contributed to discussions. AK conceived the project idea, supervised the project, analysed and interpreted data, contributed to discussions and wrote the manuscript. All authors revised and edited the manuscript, and approved the version to be published.

$\mathrm{AK}$ is the guarantor of this work and had full access to all of the data, and takes full responsibility for the integrity of the data and the accuracy of the data analysis.

\section{References}

1. Meyre D, Delplanque J, Chèvre JC et al (2009) Genome-wide association study for early-onset and morbid adult obesity identifies three new risk loci in European populations. Nat Genet 41:157-159

2. Thorleifsson G, Walters GB, Gudbjartsson DF et al (2009) Genome-wide association yields new sequence variants at seven loci that associate with measures of obesity. Nat Genet 41:18-24

3. Willer CJ, Speliotes EK, Loos RJ et al (2009) Six new loci associated with body mass index highlight a neuronal influence on body weight regulation. Nat Genet 41:25-34

4. Cypess AM, Lehman S, Williams G et al (2009) Identification and importance of brown adipose tissue in adult humans. N Engl J Med 360:1509-1517

5. Ichimura A, Hirasawa A, Poulain-Godefroy O et al (2012) Dysfunction of lipid sensor GPR120 leads to obesity in both mouse and human. Nature 483:350-354

6. Greenblum S, Turnbaugh PJ, Borenstein E (2012) Metagenomic systems biology of the human gut microbiome reveals topological shifts associated with obesity and inflammatory bowel disease. Proc Natl Acad Sci USA 109:594-599

7. Cristancho AG, Lazar MA (2011) Forming functional fat: a growing understanding of adipocyte differentiation. Nat Rev Mol Cell Biol 12:722-734

8. Wabitsch M, Brenner RE, Melzner I et al (2001) Characterization of a human pre-adipocyte cell strain with high capacity for adipose differentiation. Int J Obes Relat Metab Disord 25:8-15

9. Körner A, Wabitsch M, Seidel B et al (2005) Adiponectin expression in humans is dependent on differentiation of adipocytes and down-regulated by humoral serum components of high molecular weight. Biochem Biophys Res Commun 337:540-550

10. Gagnon A, Sorisky A (1998) The effect of glucose concentration on insulin-induced 3 T3-L1 adipose cell differentiation. Obes Res 6:157-163

11. Smith PJ, Wise LS, Berkowitz R, Wan C, Rubin CS (1988) Insulin-like growth factor-I is an essential regulator of the differentiation of 3 T3-L1 adipocytes. J Biol Chem 263:9402-9408

12. Zilberfarb V, Siquier K, Strosberg AD, Issad T (2001) Effect of dexamethasone on adipocyte differentiation markers and tumour necrosis factor-alpha expression in human PAZ6 cells. Diabetologia 44:377-386

13. Burns TW, Langley PE, Terry BE et al (1981) Pharmacological characterizations of adrenergic receptors in human adipocytes. J Clin Invest 67:467-475 
14. Kloting N, Graham TE, Berndt J et al (2007) Serum retinolbinding protein is more highly expressed in visceral than in subcutaneous adipose tissue and is a marker of intra-abdominal fat mass. Cell Metab 6:79-87

15. Heard-Costa NL, Zillikens MC, Monda KL et al (2009) NRXN3 is a novel locus for waist circumference: a genome-wide association study from the CHARGE Consortium. PLoS Genet 5:e1000539

16. Lindgren CM, Heid IM, Randall JC et al (2009) Genome-wide association scan meta-analysis identifies three loci influencing adiposity and fat distribution. PLoS Genet 5:e1000508

17. Renström F, Payne F, Nordström A et al (2009) Replication and extension of genome-wide association study results for obesity in 4923 adults from northern Sweden. Hum Mol Genet 18:1489-1496

18. Friebe D, Löffler D, Schönberg M et al (2011) Impact of metabolic regulators on the expression of the obesity associated genes FTO and NAMPT in human pre-adipocytes and adipocytes. PLoS One 6:e19526

19. Boissel S, Reish O, Proulx K et al (2009) Loss-of-function mutation in the dioxygenase-encoding FTO gene causes severe growth retardation and multiple malformations. Am J Hum Genet 85:106-111

20. Fischer J, Koch L, Emmerling C et al (2009) Inactivation of the Fto gene protects from obesity. Nature 458:894-898

21. Mountjoy KG, Mortrud MT, Low MJ, Simerly RB, Cone RD (1994) Localization of the melanocortin-4 receptor (MC4-R) in neuroendocrine and autonomic control circuits in the brain. Mol Endocrinol 8:1298-1308

22. Huszar D, Lynch CA, Fairchild-Huntress V et al (1997) Targeted disruption of the melanocortin-4 receptor results in obesity in mice. Cell 88:131-141

23. Siersbaek R, Nielsen R, Mandrup S (2010) PPARgamma in adipocyte differentiation and metabolism-novel insights from genome-wide studies. FEBS Lett 584:3242-3249

24. Berndt J, Klöting N, Kralisch S et al (2005) Plasma visfatin concentrations and fat depot-specific mRNA expression in humans. Diabetes 54:2911-2916

25. Jurvansuu J, Zhao Y, Leung DSY et al (2008) Transmembrane protein 18 enhances the tropism of neural stem cells for glioma cells. Cancer Res 68:4614-4622

26. Scherag A, Dina C, Hinney A et al (2010) Two new loci for bodyweight regulation identified in a joint analysis of genome-wide association studies for early-onset extreme obesity in French and German study groups. PLoS Genet 6:e1000916

27. Speliotes EK, Willer CJ, Berndt SI et al (2010) Association analyses of 249,796 individuals reveal 18 new loci associated with body mass index. Nat Genet 42:937-948

28. Nagahara AH, Tuszynski MH (2011) Potential therapeutic uses of BDNF in neurological and psychiatric disorders. Nat Rev Drug Discov 10:209-219

29. Cordeira J, Rios M (2011) Weighing in the role of BDNF in the central control of eating behavior. Mol Neurobiol 44:441-448

30. Xu B, Goulding EH, Zang K et al (2003) Brain-derived neurotrophic factor regulates energy balance downstream of melanocortin-4 receptor. Nat Neurosci 6:736-742

31. Unger TJ, Calderon GA, Bradley LC, Sena-Esteves M, Rios M (2007) Selective deletion of Bdnf in the ventromedial and dorsomedial hypothalamus of adult mice results in hyperphagic behavior and obesity. J Neurosci 27:14265-14274

32. El Gharbawy AH, Adler-Wailes DC, Mirch MC et al (2006) Serum brain-derived neurotrophic factor concentrations in lean and overweight children and adolescents. J Clin Endocrinol Metab 91:3548-3552

33. Krabbe KS, Nielsen AR, Krogh-Madsen R et al (2007) Brainderived neurotrophic factor (BDNF) and type 2 diabetes. Diabetologia 50:431-438

34. Kernie SG, Liebl DJ, Parada LF (2000) BDNF regulates eating behavior and locomotor activity in mice. EMBO J 19:1290-1300

35. Zaltsman Y, Shachnai L, Yivgi-Ohana N et al (2010) MTCH2/ MIMP is a major facilitator of tBID recruitment to mitochondria. Nat Cell Biol 12:553-562

36. Kulyté A, Rydén M, Mejhert N et al (2011) MTCH2 in human white adipose tissue and obesity. J Clin Endocrinol Metab 96: E1661-E1165

37. Gutierrez-Aguilar R, Kim DH, Woods SC, Seeley RJ (2012) Expression of new loci associated with obesity in diet-induced obese rats: from genetics to physiology. Obes (Silver Spring) 20:306-312

38. Hamm JK, el Jack AK, Pilch PF, Farmer SR (1999) Role of PPAR gamma in regulating adipocyte differentiation and insulinresponsive glucose uptake. Ann NY Acad Sci 892:134-145

39. Vidal-Puig AJ, Considine RV, Jimenez-Linan M et al (1997) Peroxisome proliferator-activated receptor gene expression in human tissues. Effects of obesity, weight loss, and regulation by insulin and glucocorticoids. J Clin Invest 99:2416-2422

40. Funatsu N, Miyata S, Kumanogoh H et al (1999) Characterization of a novel rat brain glycosylphosphatidylinositol-anchored protein (Kilon), a member of the IgLON cell adhesion molecule family. J Biol Chem 274:8224-8230

41. Hashimoto T, Yamada M, Maekawa S, Nakashima T, Miyata S (2008) IgLON cell adhesion molecule Kilon is a crucial modulator for synapse number in hippocampal neurons. Brain Res 1224:1-11

42. Walley AJ, Jacobson P, Falchi M et al (2012) Differential coexpression analysis of obesity-associated networks in human subcutaneous adipose tissue. Int $\mathrm{J}$ Obes (Lond) 36:137-147

43. Nishikawa K, Nakashima T, Takeda $\mathrm{S}$ et al (2010) Maf promotes osteoblast differentiation in mice by mediating the age-related switch in mesenchymal cell differentiation. J Clin Invest 120:3455-3465

44. Ren D, Li M, Duan C, Rui L (2005) Identification of SH2-B as a key regulator of leptin sensitivity, energy balance, and body weight in mice. Cell Metabol 2:95-104

45. Duan C, Yang H, White MF, Rui L (2004) Disruption of the SH2$\mathrm{B}$ gene causes age-dependent insulin resistance and glucose intolerance. Mol Cell Biol 24:7435-7443

46. Ren D, Zhou Y, Morris D, Li M, Li Z, Rui L (2007) Neuronal SH2B1 is essential for controlling energy and glucose homeostasis. J Clin Invest 117:397-406

47. Garver WS, Francis GA, Jelinek D et al (2007) The National Niemann-Pick $\mathrm{C} 1$ disease database: report of clinical features and health problems. Am J Med Genet A 143A:1204-1211

48. Jelinek D, Millward V, Birdi A, Trouard TP, Heidenreich RA, Garver WS (2011) Npc1 haploinsufficiency promotes weight gain and metabolic features associated with insulin resistance. Hum Mol Genet 20:312-321

49. Veyrieras JB, Kudaravalli S, Kim SY et al (2008) High-resolution mapping of expression-QTLs yields insight into human gene regulation. PLoS Genet 4:e1000214

50. Park KW, Waki H, Choi SP, Park KM, Tontonoz P (2010) The small molecule phenamil is a modulator of adipocyte differentiation and PPARgamma expression. J Lipid Res 51:2775-2784 